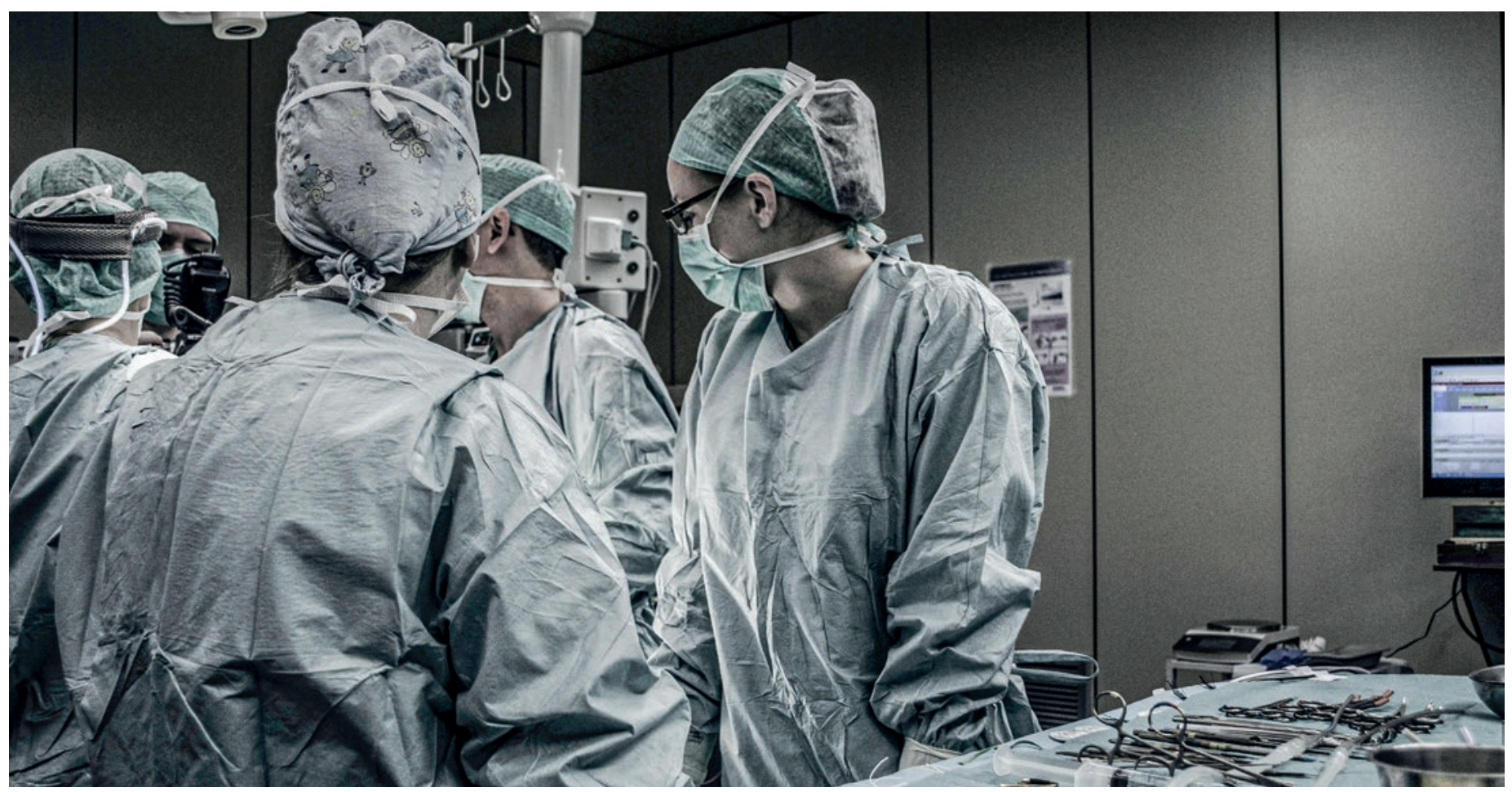

\title{
Ethique clinique en Suisse: stagnation avant la pandémie?
}

\section{Anna Zentnera, Rouven Porz ${ }^{b}$, Sibylle Ackermann ${ }^{c}$, Ralf J. Jox ${ }^{d}$}

${ }^{a}$ Cand. Dr méd., Ethique médicale et formation médicale postgraduée, Direction médecine, Insel Gruppe, Inselspital, Berne; b Prof. Dr phil, dipl. biol. AdL, Ethique médicale et formation médicale postgraduée, Direction médecine, Insel Gruppe, Inselspital, Berne; ${ }^{c}$ lic. théol., dipl. biol., Responsable du ressort Ethique, ASSM, Berne; d Prof. Dr méd. Dr phil., Unité d'éthique clinique, Institut des humanités en médecine, CHUV et Université Lausanne

Léthique clinique offre aux professionnels de la santé un soutien dans la pesée de valeurs complexes, la définition de processus de prises de décisions éthiques, le conseil sur des questions d'éthique institutionnelle, mais également dans l'organisation de la formation continue et de l'enseignement universitaire en éthique. L'Académie Suisse des Sciences Médicales (ASSM) soutient le développement de cette discipline jeune et évalue régulièrement la situation. Les résultats de la quatrième enquête fournissent un bilan intermédiaire avant la pandémie de Covid-19.

Ces vingt dernières années, la discipline de l'éthique clinique s'est constamment développée et professionnalisée en Suisse [1-3]. Depuis près de vingt ans, l'ASSM - entre autres - soutient son développement. Elle recommande l'instauration et le recours à une structure de soutien éthique en médecine dans un grand nombre de ses directives [4]. Les trois enquêtes transversales menées par l'ASSM ont montré qu'au fil des années, le nombre de structures d'éthique clinique explicites dans les hôpitaux suisses a augmenté de 18\% en 2002 à $44 \%$ en 2006 et à $48 \%$ en 2014 (cf. [1-3]). Les trois premières enquêtes ont montré que, du point de vue thématique, le domaine d'activité de l'éthique clinique a évolué au fil du temps, mais dans ces mêmes enquêtes, les questions éthiques les plus fréquemment soumises à la structure d'éthique concernaient l'«interruption d'un traitement». Les autres questions portaient principalement sur la «réanimation» et l'«assistance au dé- 
cès» (2002), les problèmes soulevés par les «mesures de contrainte» (2006) et par les «directives anticipées» (2014). L'enquête de 2014 montre également que les conflits de valeurs au sein de l'équipe de soins sont devenus un sujet de plus en plus important, tout comme les thèmes à forte composante juridique tels que l'évaluation de la capacité de discernement des patientes et des patients.

\section{Enquête sans lien avec la pandémie}

La quatrième enquête de l'ASSM a été réalisée juste avant la pandémie de Covid-19, pendant l'hiver 2019/2020. Les données n'incluent donc aucune question d'éthique spécifique au Covid-19, comme par exemple celles concernant le triage des patientes et des patients dans les unités de soins intensifs ou des situations de soins moralement difficiles dans les EMS.

A l'instar des enquêtes précédentes, celle-ci s'est à nouveau déroulée en deux étapes. Dans un premier temps, tous les hôpitaux de soins aigus, les cliniques psychia-

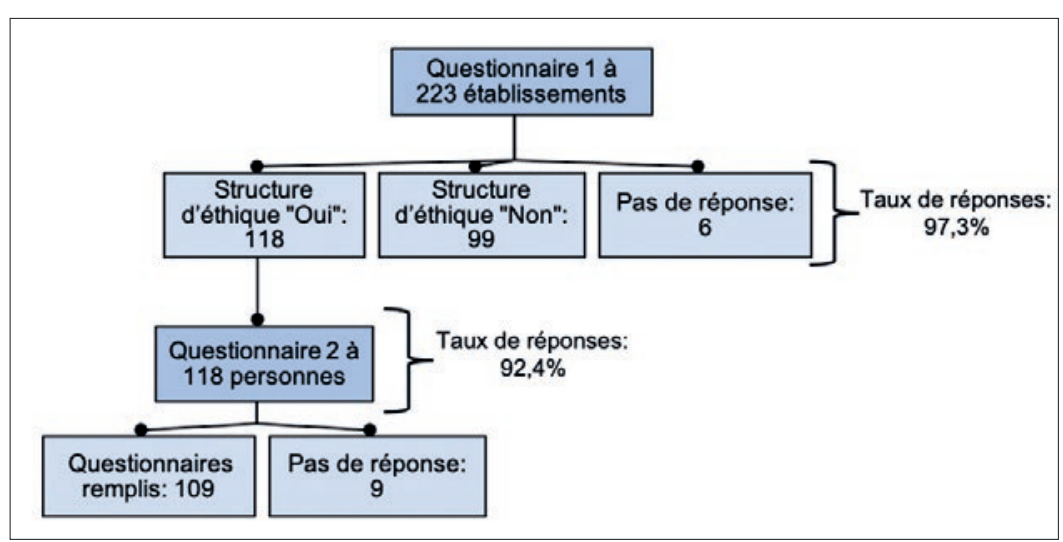

Figure 1: Méthodologie et taux de réponses aux questionnaires 1 et 2 de l'enquête nationale de I'ASSM sur le nombre de structures d'éthique en 2020.

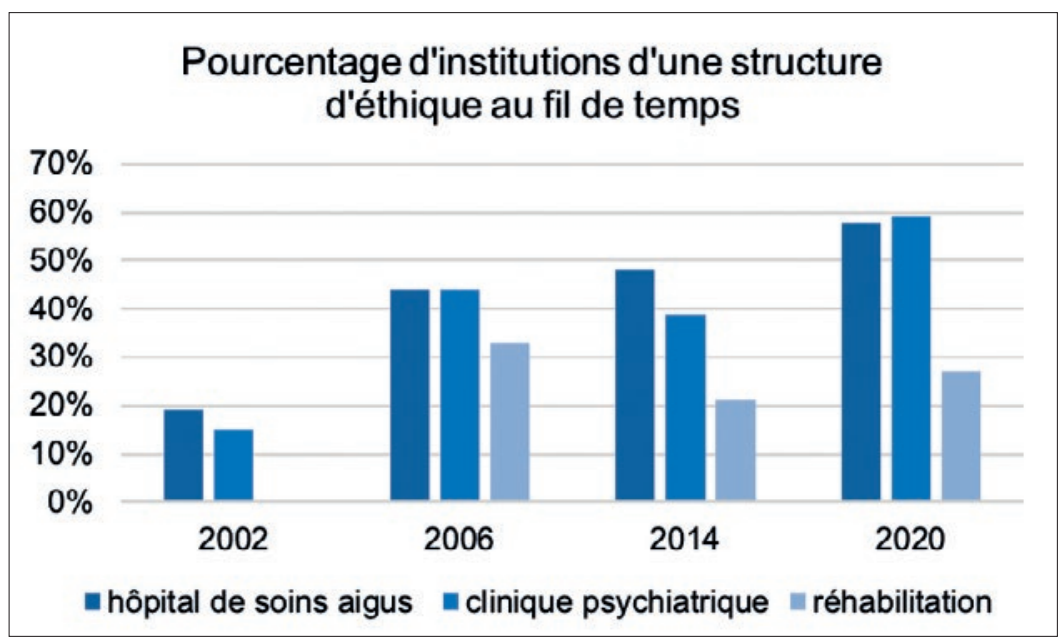

Figure 2: Pourcentage des hôpitaux dotés de structures d'éthique, répartis selon le type d'hôpital, dans les enquêtes de 2002, 2006, 2014 et 2020. triques et les cliniques de réhabilitation qui figurent dans la liste de l'association nationale des hôpitaux $\mathrm{H}+$ ont été interrogés (en tout 223 institutions) quant à l'existence d'une structure de soutien éthique dans leur établissement, avec un premier résultat important: actuellement, en chiffres absolus, 50,2\% de ces institutions (118 institutions) disposent d'une structure d'éthique clinique explicite. Ces chiffres sont présentés ci-dessous dans la figure 1. Force est de constater que le nombre de structures d'éthique en Suisse n'a pas connu d'augmentation majeure depuis l'enquête de 2014 (48\%; cf. introduction ci-dessus).

Dans un deuxième temps, un questionnaire en ligne détaillé a été adressé aux 118 institutions qui disposent d'une structure d'éthique. Le taux de réponses s'élevait à $92,4 \%$. Sur la base de ces données, nous présentons (a) la répartition des structures d'éthique dans les différents types d'institutions au cours des vingt dernières années. Nous nous concentrons ensuite sur (b) les responsables de l'éthique dans les institutions, (c) la composition des structures d'éthique, (d) le contenu des activités des structures d'éthique et (e) les principaux thèmes éthiques traités.

\section{(a) Répartition des structures d'éthique}

Si l'on considère le pourcentage des hôpitaux suisses dotés de structures d'éthique (fig. 2), on constate qu'en 2020, plus de la moitié des hôpitaux de soins aigus et des unités psychiatriques disposent de telles structures. De même, de plus en plus de cliniques de réhabilitation s'engagent, elles aussi, dans l'éthique clinique. Toutefois, on constate dans le même temps - comme mentionné ci-dessus en chiffres absolus - qu'en ce qui concerne les hôpitaux aigus, aucune augmentation majeure du nombre de structures de soutien éthique ne peut être observée récemment (par rapport à 2002 et 2006). C'est entre 2002 et 2006 que les structures d'éthique clinique dans les hôpitaux de soins aigus ont connu une forte expansion.

\section{(b) Responsables de l'éthique dans les institutions} Qui sont les responsables de l'éthique clinique dans les institutions et quelle est leur formation en éthique? La Suisse présente, à cet égard, un tableau mitigé. Si certaines de ces personnes disposent d'une formation pré- et postgraduée, d'autres n'ont suivi aucune formation. Traduit en chiffres (fig. 3): 30\% des répondants ont indiqué avoir suivi une formation pré- et postgraduée spécifique en éthique (par ex. doctorat, master ou Certificate of Advanced Studies). Presque autant des personnes interrogées (29\%) ont suivi plusieurs formations ou ont étudié l'éthique comme discipline annexe pendant leur formation de base. $18 \%$ des répondants ne 


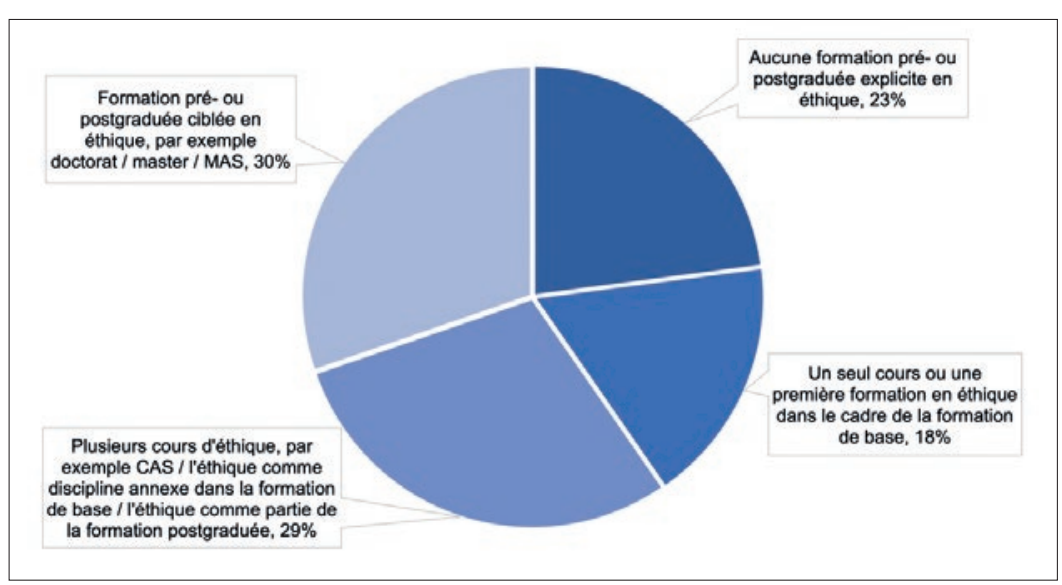

Figure 3: Présentation de la formation pré- et postgraduée en éthique des responsables de l'éthique dans les différentes institutions.

disposent que d'une formation minimale en éthique, par exemple une participation à un seul cours d'éthique. Presque un quart (23\%) des responsables d'une structure d'éthique clinique ont indiqué n'avoir suivi aucune formation en éthique. Dès lors, une réflexion sur des nouvelles possibilités de formation professionnelle en éthique clinique s'impose en Suisse.

\section{(c) Compositions des structures d'éthique}

La figure 4 montre que dans $69 \%$ des hôpitaux avec des offres en éthique, la structure d'éthique est un "comité d'éthique» (nommé également "commission d'éthique», "conseil d'éthique» ou "forum d'éthique») et que $27 \%$ de ces hôpitaux disposent d'experts en éthique engagés - à divers degrés - par l'institution pour des missions d'éthique clinique. Egalement $27 \%$ de ces hôpitaux ont recours à un service de conseil éthique fourni par une personne externe à l'institution. En comparaison avec l'enquête de 2014, le nombre de comités d'éthique a clairement diminué, celui des professionnels de l'éthique employés dans une institution n’a que

\begin{tabular}{|c|c|c|c|}
\hline $\begin{array}{l}\text { Groupes professionnels } \\
\text { représentés }\end{array}$ & $\begin{array}{l}\text { \% des structures } \\
\text { d'éthique }\end{array}$ & Direction & $\begin{array}{l}\text { \% des structures } \\
\text { d'éthique }\end{array}$ \\
\hline Corps médical & 95 & Multiprofessionnelle & 55 \\
\hline Soins infirmiers & 90 & Monoprofessionnelle & 33 \\
\hline Ethique & 70 & Corps médical & 44 \\
\hline $\begin{array}{l}\text { Assistance spirituelle/ } \\
\text { théologie }\end{array}$ & 70 & Ethique & 32 \\
\hline Droit & 48 & Soins infirmiers & 10 \\
\hline Administration/direction & 45 & Droit & 5 \\
\hline Psychologie & 41 & & \\
\hline Assistance sociale & 29 & & \\
\hline $\begin{array}{l}\text { Physiothérapie/ergo- } \\
\text { thérapie }\end{array}$ & 26 & & \\
\hline Sage-femmes & 16 & & \\
\hline
\end{tabular}

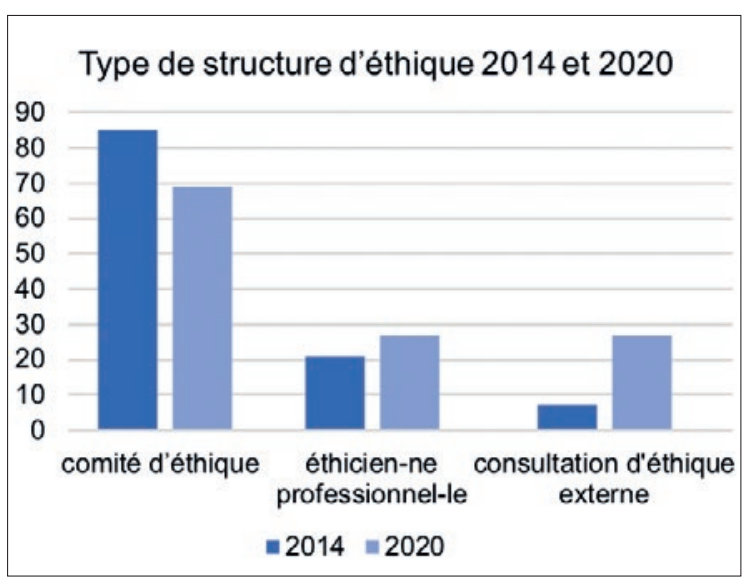

Figure 4: Différents types de structures d'éthique en hôpital en 2014 et 2020. Réponses indiquées en pourcentage.

légèrement augmenté. En revanche, le nombre de consultations d'experts externes a quadruplé.

Les structures d'éthique comprennent presque toujours des représentants du corps médical et des soins infirmiers, souvent également des éthiciennes ainsi que des représentants spirituels (théologiens ou assistants spirituels), plus rarement des juristes, des représentantes de l'administration ou de la direction des hôpitaux et d'autres professions de la santé. Ces résultats sont présentés dans le tableau 1. La plupart du temps, la direction de la structure d'éthique est multiprofessionnelle, sachant que les représentants du corps médical et de l'éthique sont majoritaires par rapport au personnel infirmier et aux juristes. Une formation pré- ou postgraduée des membres n'est exigée que dans $38 \%$ des structures d'éthique. Les structures d'éthique pourraient gagner en professionnalisme si une formation pré- ou postgraduée était exigée. Des offres correspondantes doivent être créées ou développées.

\section{(d) Contenus des activités}

Conformément aux indications des responsables, les structures d'éthique qui ont participé à l'enquête se chargent de tâches multiples dans le domaine de l'éthique clinique, comprenant entre autres les activités classiques de conseil dans les cas individuels (par exemple dans des discussions de cas éthiques), l'élaboration de directives et/ou l'organisation de formations continues en éthique (cf. fig. 5).

Il convient de noter que plus de $60 \%$ des répondants considèrent que leur mission englobe aussi les prises de décisions occasionnelles dans des cas individuels $(52 \%$ en 2014). Il s'agit là d'un point délicat et l'ASSM souligne expressément depuis 2012 que la responsabilité de la décision revient toujours au médecin et à l'équipe de soins (ainsi qu'au patient) et ne relève pas du domaine de compétence de la structure d'éthique [5]. 


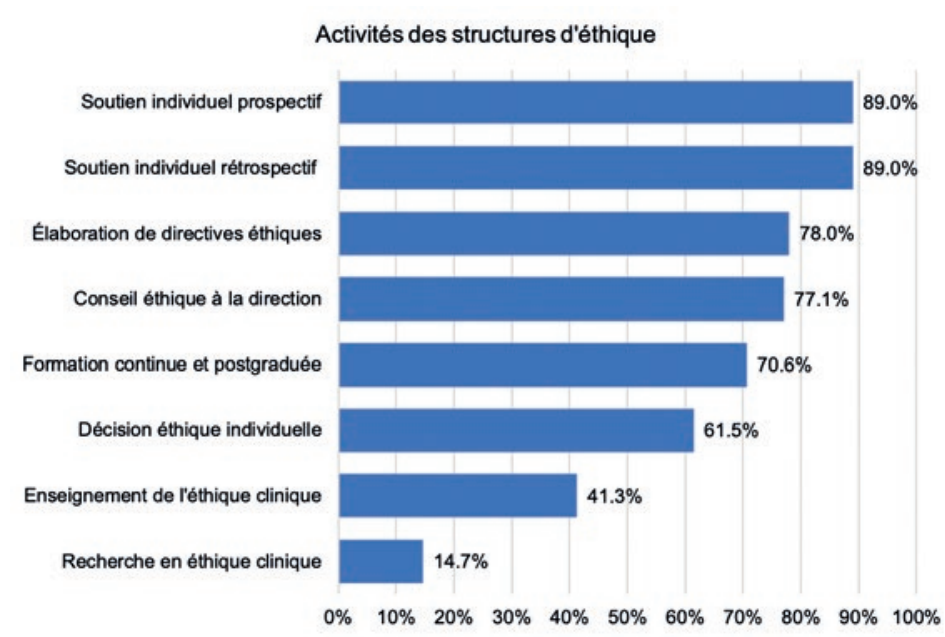

Figure 5: Activités des structures d'éthique selon les indications des responsables de l'éthique (en pourcentage des réponses, plusieurs réponses possibles).

A la question de savoir si les structures d'éthique documentent leurs activités, $80 \%$ des personnes interrogées ont répondu par l'affirmative, $14 \%$ par la négative (6\% d'abstention). Par ailleurs, un peu plus d'un tiers des structures d'éthique utilisent des outils pour évaluer l'impact de leurs activités.

\section{(e) Thèmes éthiques}

Les responsables de l'éthique ont également été interrogés sur la fréquence des différents thèmes et problématiques soumis à leur structure d'éthique. On constate à cet égard que les thèmes éthiques classiques et transversaux qui surviennent dans tous les domaines de la médecine sont particulièrement représentés, tels que la réflexion quant à un changement d'objectif thérapeutique, respectivement à l'interruption d'un traitement, la prise en compte de directives anticipées et les questions éthiques relatives à l'évaluation de la capacité de discernement (cf. fig. 6).

En revanche, les résultats de l'enquête montrent que les thèmes éthiques en vogue qui font les grands titres dans les médias (intelligence artificielle, numérisation, génétique, médecine reproductive, transplantation) ne sont que rarement traités dans les structures d'éthique clinique.

Globalement, ces vingt dernières années, l'éthique clinique a beaucoup évolué en Suisse. Toutefois, les résultats de la quatrième enquête de l'ASSM de 2020 révèlent encore des lacunes. Il semble que le développement de structures d'éthique stagne; nombreuses sont les institutions - même grandes - qui ne disposent toujours pas d'une structure d'éthique. Certaines structures d'éthique établies considèrent encore que, contrairement aux recommandations nationales, leur rôle consiste à prendre elles-mêmes des décisions. En Suisse, de nombreux responsables de l'éthique se heurtent toujours à la difficulté de suivre des formations spécifiques en éthique clinique. La Suisse n'est pas seule dans ce cas; Ellen Fox, par exemple, est arrivée aux mêmes conclusions dans son

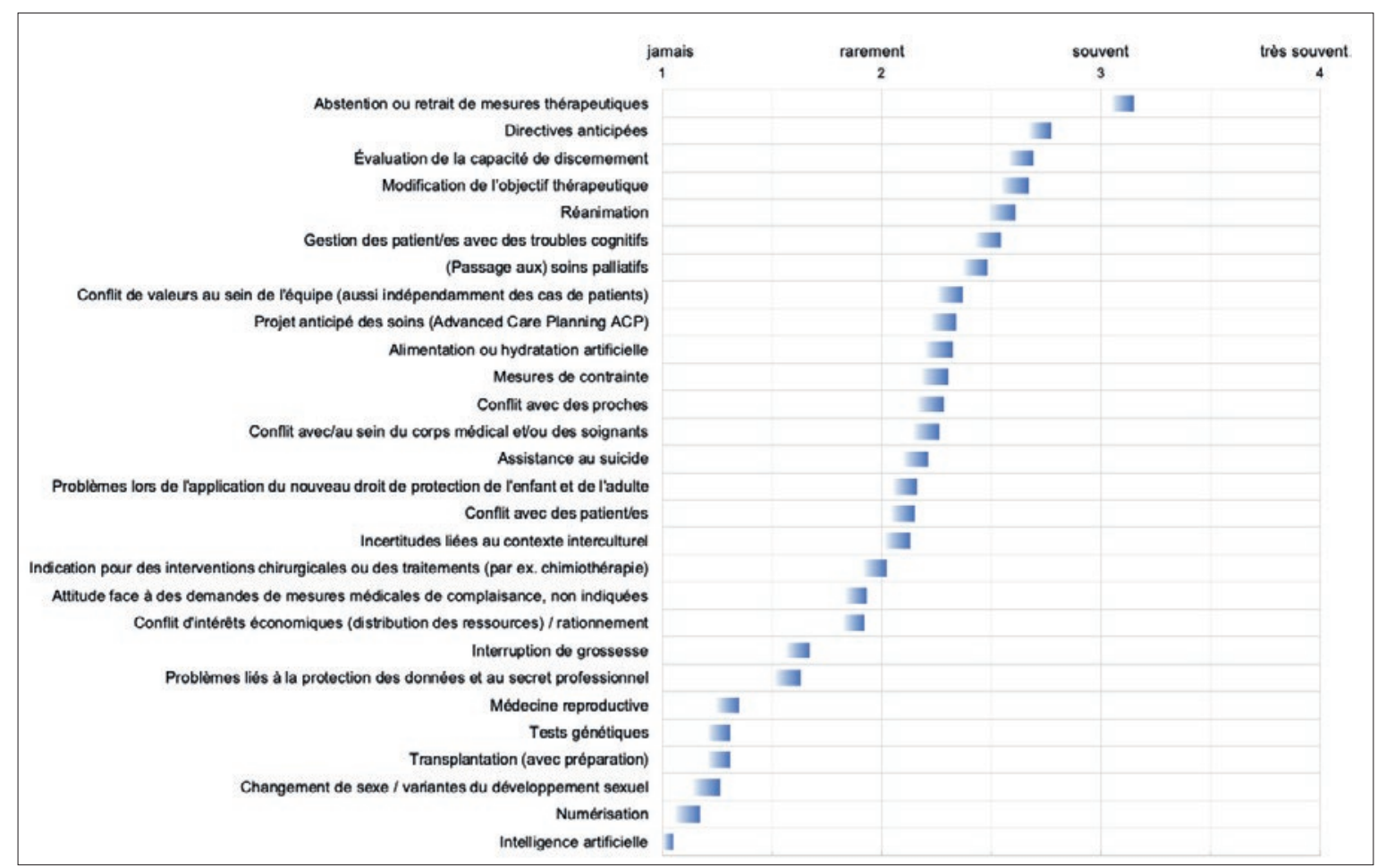

Figure 6: Fréquence à laquelle certains thèmes et problématiques éthiques sont traités dans la structure d'éthique respective. 
étude de suivi concernant l'évolution de l'éthique clinique aux Etats-Unis (comparaison entre 2000 et 2020). Elle aussi souligne que de nombreux responsables de l'éthique n'ont pas suivi de formation pré- ou postgraduée directe en éthique. Elle constate, en outre, qu'il existe des différences importantes entre les petits hôpitaux périphériques et les grandes institutions académiques [6].

Il convient à présent de souligner que les enjeux éthiques liés à la pandémie de Covid-19 pourraient avoir un impact majeur sur l'évolution de la discipline de l'éthique clinique. Ainsi, les résultats de la quatrième enquête offrent non seulement un aperçu fondé de la situation au niveau national, mais constituent également une solide base de comparaison pour la prochaine enquête. Il sera intéressant de voir de quelle manière la pandémie aura eu un impact sur les structures d'éthique nationales.

Cet article est basé sur des extraits des résultats de la quatrième enquête. Vous trouverez tous les détails sur le site web de l'ASSM: assm.ch/soutien-ethique/etudes

\section{L'essentiel en bref}

- La quatrième enquête de I'ASSM a été réalisée juste avan la pandémie de Covid-19, pendant I'hiver 2019/2020. Les données n'incluent aucune question d'éthique spécifique au Covid-19.

Sibylle Ackermann Responsable du ressort Ethique

Académie Suisse des Sciences Médicales ethics[at]samw.ch
- En 2020, plus de la moitié des hôpitaux de soins aigus et des unités psychiatriques disposaient de structures d'éthique.

- Le développement de structures d'éthique stagne: de nombreuses institutions - même grandes - ne sont toujours pas dotées d'une structure d'éthique.

\section{Recommandations de I'ASSM}

L'éthique clinique est une discipline relativement jeune. C'est pourquoi, ces dernières années, I'ASSM a formulé des recommandations nationales sur la manière de pratiquer concrètement l'éthique clinique et de l'enseigner dans les hautes écoles et les universités. Les recommandations "Soutien éthique en médecine» publiées en 2012 par I'ASSM renforcent la prise de conscience de l'éthique et permettent de comprendre comment l'éthique clinique peut aider à prendre des décisions cliniques responsables et à éviter les conflits d'intérêts [4]. Les recommandations "Enseignement de l'éthique aux professions de la santé», publiées en 2019 [7], mettent l'accent sur la formation et proposent un ensemble de thèmes et de contenus destinés à soutenir l'enseignement de l'éthique dans sa globalité, c'est-àdire les méthodes, les attitudes et les connaissances nécessaires.

\section{Crédits photo}

Piron Guillaume / Unsplash

\section{Références}

1 ASSM. Salathé M, Leuthold M, Amstad H, Valloton M. Les comités d'éthique clinique en Suisse - un état des lieux. Bull Med Suisses. 2003;84(50):2682-5.

2 SAMW. Salathé $M$, Amstad H, Jünger M, Leuthold M, Regamey C. Institutionalisierung der Ethikberatung an Akutspitälern, psychiatrischen Kliniken, Pflegeheimen und Einrichtungen der Rehabilitation der Schweiz: Zweite Umfrage der Schweizerischen Akademie der Medizinischen Wissenschaften. Bioethica Forum. 2008;1(1):8-14.

3 SAMW. Ackermann S, Balsiger L, Salathé M. Ethikstrukturen an Akutspitälern, Psychiatrischen Kliniken und Rehabilitationskliniken der Schweiz. Bioethica Forum. 2014;9(2):52-9.

4 ASSM. 2012. Soutien éthique en médecine. Recommendations medico-éthiques. Téléchargement: assm.ch/directives

5 Cf. [4], page 7: «La responsabilité des décisions reste auprès du médecin et de l'équipe soignante».

6 Fox E, Danis M, Tarzian A, Duke C. Ethics Consultation in U.S. Hospitals: A National Follow-Up Study. In: American Journal of Bioethics. 2021;26;1-14. doi: 10.1080/15265161.2021.1893547

7 ASSM. 2019. Enseignement de l'éthique aux professions de la santé. Téléchargement: assm.ch/directives 